\title{
A MIN-MAX PRINCIPLE WITH A RELAXED BOUNDARY CONDITION
}

\author{
N. GHOUSSOUB
}

(Communicated by William J. Davis)

\begin{abstract}
A standard Min-Max procedure to find critical points for a $C^{1}$ functional $\varphi$ verifying a compactness condition of Palais-Smale type on a smooth Banach manifold $X$ consists of finding an appropriate class $\mathscr{F}$ of compact subsets of $X$, all containing a fixed boundary $B$, and then showing that the value $c=\inf _{A \in \mathscr{F}} \sup _{x \in A} \varphi(x)$ is a critical level, provided it satisfies $\sup \varphi(B)<c$. In this paper, we refine this procedure by relaxing the boundary condition.
\end{abstract}

\section{INTRODUCTION}

Let $\varphi$ be a $C^{1}$-functional verifying a compactness condition of Palais-Smale type on a smooth Banach manifold $X$. A well-established procedure for exhibiting critical points for $\varphi$ consists of finding an appropriate class $\mathscr{F}$ of compact subsets of $X$, all containing a fixed boundary $B$, and then showing that the number

$$
c=c(\varphi, \mathscr{F})=\inf _{A \in \mathscr{F}} \sup _{x \in A} \varphi(x)
$$

is actually a critical value for $\varphi$ provided it satisfies

$$
\sup \varphi(B)<c .
$$

Our goal in this note is to relax the above boundary condition in three ways. First, we shall consider the case where $B$ is not necessarily compact, nor contained in all the members of the class $\mathscr{F}$ and for that, we introduce the following

Definition 1. Let $B$ be a closed subset of $X$. Say that a class $\mathscr{F}$ of compact subsets of $X$ is a homotopy-stable family with extended boundary $B$ if for any set $A$ in $\mathscr{F}$ and any $\eta \in C([0,1] \times X ; X)$ verifying $\eta(t, x)=x$ for all $(t, x)$ in $(\{0\} \times X) \cup([0,1] \times B)$ we have that $\eta(\{1\} \times A) \in \mathscr{F}$.

In the second improvement, we will allow $\sup _{B} \varphi$ to take the critical value $c$. Recall that in the classical theorem, the critical point is obtained by taking a point that maximizes $\varphi$ on an "optimal path", that is, a set $A \in \mathscr{F}$ on which $\sup \varphi(A)$ is essentially equal to $c$. Condition (F0) makes sure that such a point

Received by the editors May 5, 1990 and, in revised form, June 11, 1991.

1991 Mathematics Subject Classification. Primary 35J65, 47H15, 49B27. 
is not on the boundary $B$. In general, not every point that maximizes $\varphi$ on $A$ is necessarily a critical point, however, we will be able to prove an existence result by merely assuming that not all highest points on a given path $A$ occur on the boundary, in other words,

$$
M(A ; \varphi) \backslash B \neq \varnothing \quad \text { for every } A \in \mathscr{F},
$$

where $M(A ; \varphi)=\{x \in A ; \varphi(x)=\sup \varphi(A)\}$. Since necessarily $\sup \varphi(B) \leq$ $c$ (when $B$ is contained in all the members of $\mathscr{F}$ ), this new condition is equivalent to

$$
A \cap\{\varphi \geq c\} \backslash B \neq \varnothing \quad \text { for every } A \in \mathscr{F} .
$$

This result was obtained recently by Brezis-Nirenberg in [BN].

For the third improvement, we recall that in [G1], the boundary condition was relaxed in the presence of a dual set in the following fashion: We suppose the existence of a closed subset $F$ of $X$ such that

(F1) $\quad F \cap B=\varnothing$ and $F \cap A \neq \varnothing$ for all $A \in \mathscr{F}$ (i.e., $B$ and $F$ link via $\mathscr{F}$ ) and

$$
\inf \varphi(F) \geq c .
$$

We then prove (assuming again that $B$ is contained in all members of $\mathscr{F}$ ) the existence of a critical point for $\varphi$ at the level $c$ and on the set $F$. The "classical condition" (F0) means that the set $F=\{\varphi \geq c\}$ verifies (F1) and (F2). Various other more interesting choices of smaller sets $F$ are possible, and we refer to [G1, G2] for some examples.

In this note, we prove an existence result that combines all these refinements. We do not know of an example where the classical results have already failed and where the following theorem can be applied. However, we believe that its proof is another interesting illustration of the usefulness of the perturbation methods initiated in [GP].

Theorem 1. Let $\varphi$ be a $C^{1}$-functional on a complete connected $C^{1}$-Finsler manifold $X$ and consider a homotopy-stable family $\mathscr{F}$ with an extended closed boundary $B$. Set $c=c(\varphi, \mathscr{F})$, and let $F$ be a closed subset of $X$ verifying

$$
A \cap F \backslash B \neq \varnothing \quad \text { for every } A \in \mathscr{F}
$$

and

$$
\sup \varphi(B) \leq c \leq \inf \varphi(F) .
$$

Then, for any sequence of sets $\left(A_{n}\right)_{n}$ in $\mathscr{F}$ such that $\lim _{n} \sup \varphi\left(A_{n}\right)=c$, there exists a sequence $\left(x_{n}\right)_{n}$ in $X \backslash B$ such that

(i) $\lim _{n} \varphi\left(x_{n}\right)=c$;

(ii) $\lim _{n}\left\|d \varphi\left(x_{n}\right)\right\|=0$;

(iii) $\lim _{n} \operatorname{dist}\left(x_{n}, F\right)=0$;

(iv) $\lim _{n} \operatorname{dist}\left(x_{n}, A_{n}\right)=0$.

The function $\varphi$ is said to verify the Palais-Smale condition at the level $c$ and around the set $F$ (in short $(P S)_{F, c}$ ) if any sequence $\left(x_{n}\right)_{n}$ in $X$ verifying $\lim _{n} \varphi\left(x_{n}\right)=c, \lim _{n}\left\|d \varphi\left(x_{n}\right)\right\|=0$, and $\lim _{n} \operatorname{dist}\left(x_{n}, F\right)=0$ has a convergent subsequence.

As customary, $K_{c}$ will denote the set $\{x \in X ; \varphi(x)=c, d \varphi(x)=0\}$. The following is now immediate. 
Theorem 1.bis. Under the hypothesis of Theorem 1 and assuming that $\varphi$ verifies $(P S)_{F, c}$, we obtain that the set $F \cap K_{c}$ is nonempty.

Remark (a). Note that under condition $\left(F^{\prime} 2\right)$, the hypothesis $\left(F^{\prime} 1\right)$ is equivalent to

$$
M(A \cap F ; \varphi) \backslash B \neq \varnothing \quad \text { for every } A \in \mathscr{F} .
$$

Note also that $\sup \varphi(B) \leq c$ is automatically satisfied when $B$ is assumed to be contained in all members of $\mathscr{F}$. It is then clear that conditions $\left(F^{\prime} 1\right)$ and $\left(F^{\prime} 2\right)$ are implied by (F1) and (F2) in the case of a homotopy stable family with a real boundary $B$.

Before proceeding with the proofs we give a few immediate applications.

Corollary 1. Let $X, \varphi, B, \mathscr{F}$, and $c=c(\varphi, \mathscr{F})$ be as in Theorem 1 , and assume that $\varphi$ verifies $(P S)_{c}$. Suppose $\left(\mathrm{F}^{\prime} 0\right)$ and

$$
A \cap\{\varphi \geq c\} \backslash B \neq \varnothing \text { for every } A \in \mathscr{F}
$$

and

$$
\sup \varphi(B) \leq c
$$

Then the set $K_{c}$ is nonempty.

Proof. It is enough to apply Theorem 1.bis with the set $F=\{\varphi \geq c\}$ and the boundary $B$.

Remark (b). The above corollary applies, in particular, if

$$
A \cap\{\varphi>c\} \neq \varnothing \quad \text { for every } A \in \mathscr{F}
$$

and

$$
\sup \varphi(B) \leq c \text {. }
$$

In that case, the boundary is taken to be $B^{\prime}=\{\varphi \leq c\}$.

Corollary 2. Let $X, \varphi, B, \mathscr{F}$, and $c=c(\varphi, \mathscr{F})$ be as in Theorem 1 , and suppose there exists a closed set $F$ verifying $\left(F^{\prime} 1\right)$ and

$$
\sup \varphi(B) \leq \inf \varphi(F) \text {. }
$$

If $\varphi$ verifies $(P S)_{c}$, then the set $K_{c}$ is nonempty.

Proof. Note that hypothesis $\left(\mathrm{F}^{\prime} 1\right)$ always implies that $\inf \varphi(F) \leq c$. So either $\sup \varphi(B)<c$ or $\sup \varphi(B)=\inf \varphi(F)=c$. In both cases, condition $\left(\mathrm{F}^{\prime} 0\right)$ and $\left(F^{\prime \prime} 0\right)$ are satisfied. Corollary 1 then applies to get the claim. In the second case, a critical point can be found on $F$.

Now consider the following notation

$$
G_{c}=\{x \in X ; \varphi(x)<c\} \text { and } \varphi_{c}=\{x \in X ; \varphi(x) \leq c\} .
$$

Corollary 3. Under the hypothesis of Theorem 1 , for any $\delta>0$ and $\varepsilon>0$,

(1) there exists $A \in \mathscr{F}$ such that $\sup \varphi(A)<c+\varepsilon, \quad A \subset(X \backslash F) \cup B \cup\left(F \cap K_{c}\right)^{\delta}, \quad A \cap F \cap\left(F \cap K_{c}\right)^{\delta} \backslash B \neq \varnothing ;$

(2) there exists $A^{\prime} \in \mathscr{F}$ such that

$$
\sup \varphi\left(A^{\prime}\right)<c+\varepsilon, \quad A^{\prime} \subseteq G_{c} \cup B \cup K_{c}^{\delta}, \quad A^{\prime} \cap L_{c} \cap K_{c}^{\delta} \backslash B \neq \varnothing .
$$


Proof. (1) If not then the set $F^{\prime}=F \backslash\left(F \cap K_{c}\right)^{\delta}$ would verify $\left(\mathrm{F}^{\prime} 1\right)$ and $\left(\mathrm{F}^{\prime} 2\right)$ with respect to $\mathscr{F}$. By Theorem 1.bis, this would imply that $F^{\prime} \cap K_{c} \neq \varnothing$, which is absurd.

For (2), it is enough to apply (1) to the sets $F^{\prime}=\{\varphi \geq c\}$ and $B$.

Remark (c). Under the classical condition: $\sup \varphi(B)<c$, we obtain the wellknown result about the existence of $A \in \mathscr{F}$ with $A \subseteq G_{c} \cup K_{c}^{\delta}$.

On the other hand, under the conditions $\left(\mathrm{F}^{\prime \prime \prime} 0\right)$ and $\left(\mathrm{F}^{\prime \prime} 0\right)$, we may conclude that for any $\delta>0$ and any $\varepsilon>0$, there exists $A \in \mathscr{F}$ such that $\sup \varphi(A)<c+\varepsilon$ and $A \subseteq \varphi_{c} \cup K_{c}^{\delta}$. To see that, it is enough to apply Corollary 3 with $B=\varphi_{c}$ and $F=\{\varphi \geq c\}$.

Before proceeding with the proof of Theorem 1, we recall the various properties of Finsler manifolds and the functions defined on them that we will need in the sequel.

Let $X$ be a $C^{1}$-Banach manifold with $T(X)$ as tangent bundle and $T_{x}(X)$ as tangent space at the point $X$. Recall that a Finsler structure on $T(X)$ is a continuous function \|\|$: T(X) \rightarrow[0,+\infty)$ such that

(a) For each $x \in X$, the restriction \|\|$_{x}$ of \|\| to $T_{x}(X)$ is a norm on the latter.

(b) For each $x_{0} \in X$ and $k>1$, there is a neighborhood $U$ of $x_{0}$ such that

$$
\frac{1}{k}\|\|_{x} \leq\|\|_{x_{0}} \leq k\|\|_{x} \text { for all } x \in U .
$$

If $\sigma:[a, b] \rightarrow X$ is a $C^{1}$-path, the length of $\sigma$ is defined by $L(\sigma)=\int_{a}^{b}\|\dot{\sigma}(t)\| d t$. The distance $\rho(x, y)$ between two points $x$ and $y$ in the same connected component of $X$ is defined as the infimum of $L(\sigma)$ over al $\sigma$ joining $x$ and $y . \rho$ is then a metric on each component of $X$ (called the Finsler metric), and it is consistent with the topology of $X$.

If $\varphi \in C^{1}(X, \mathbf{R})$, the differential of $\varphi$ at $x$, denoted by $d \varphi(x)$, is an element of the cotangent space of $X$ at $x$, which is the dual space $T_{x}(X)^{*}$. The latter, equipped with the dual norm, induces a dual Finsler structure on the cotangent bundle $T(X)^{*}$.

Suppose now that $x$ is not a critical point for $\varphi$; then there exists a vector $v(x)$ in $T_{x}(X)$ such that

(c) $\|v(x)\|<2\|d \varphi(x)\|$ and $\langle v(x), d \varphi(x)\rangle>\|d \varphi(x)\|^{2}$.

Moreover, by using a suitable partition of unity, one can construct the pseudogradient vector field $v(x)$ continuously on the set of regular points of $\varphi$ (see for instance [P]).

We need the following

Lemma 1. Let $\varphi$ be a $C^{1}$-functional on a complete connected $C^{1}$-Finsler manifold $X$, and let $B$ and $C$ be two closed and disjoint subsets of $X$. Suppose that $C$ is compact and that $\|d \varphi(x)\|>2 \varepsilon>0$ for every $x \in C$. Then for each $k>1$, there exist a positive continuous function $g$ on $X$ and a deformation $\alpha \in C([0,1] \times X ; X)$ such that for some $t_{0}>0$, the following hold for every $t \in\left[0, t_{0}\right)$.

(i) $\alpha(t, x)=x$ for every $x \in B$.

(ii) $\rho(\alpha(t, x), x)) \leq k t$ for every $x \in X$.

(iii) $\varphi(\alpha(t, x))-\varphi(x) \leq-\varepsilon g(x) t$ for every $x \in X$.

(iv) $g(x)=1$ for all $x \in C$. 
Proof. Fix $k>1$, and use (a), (b), and (c) above to find for each $x_{i} \in C$, a chart $f_{i}: U_{i} \rightarrow T_{x_{i}}(X)$ and $v_{i} \in T_{x_{i}}(X)$ such that

$$
\frac{1}{k}\|\|_{x} \leq\|\|_{x_{i}} \leq k\|\|_{x} \text { for all } x \in U_{i}
$$

and

$$
\left\langle\left(\varphi \circ f_{i}^{-1}\right)^{\prime}(y), \frac{v_{i}}{\left\|v_{i}\right\|}\right\rangle \geq \frac{1}{2}\left\|d \varphi\left(x_{i}\right)\right\| \text { for all } y \in f_{i}\left(U_{i}\right)
$$

Let $V_{i} \subset U_{i}$ be an open neighborhood of $x_{i}$ such that for some $\delta_{i}>0$,

$$
B\left(V_{i}, \delta_{i}\right) \subset U_{i} \text { and } B\left(f_{i}\left(V_{i}\right), \delta_{i}\right) \subset f_{i}\left(U_{i}\right),
$$

where $B(W, \delta)$ denotes the $\delta$-neighborhood of the set $W$ in the appropriate metric.

Since $C$ is compact, we can find a finite covering $\left(V_{i}\right)_{i=1}^{m}$ to which we can associate a continuous partition of unity $\left(\chi_{i}\right)_{i=1}^{m}$. Let $l: X \rightarrow[0,1]$ be a continuous function such that

$$
l(x)= \begin{cases}1 & \text { if } x \in C, \\ 0 & \text { if } x \in\left(X \backslash \bigcup_{i=1}^{m} V_{i}\right) \cup B .\end{cases}
$$

Let $\delta_{0}=\min \left\{\delta_{1}, \ldots, \delta_{m}\right\}$ and $t_{0}=\delta_{0} /\left(1+k^{2}\right)$. Starting with $\alpha_{0}(t, x)=x$, we define by induction on $j(1 \leq j \leq m)$, the functions

$$
\alpha_{j}(t, x)= \begin{cases}f_{j}^{-1}\left(f_{j}\left(\alpha_{j-1}(t, x)\right)-t l(x) \chi_{j}(x) v_{j} /\left\|v_{j}\right\|\right) & \text { if } \alpha_{j-1}(t, x) \in U_{j}, \\ \alpha_{j-1}(t, x) \text { otherwise. } & \end{cases}
$$

We now prove that if $t \in\left(0, t_{0}\right)$, we have for each $1 \leq j \leq m$,

$$
\alpha_{j}(t, x) \text { is well defined and continuous. }
$$

(i.e., $f_{j}\left(\alpha_{j-1}(t, x)\right)-t l(x) \chi_{j}(x) v_{j} /\left\|v_{j}\right\| \in f_{j}\left(U_{j}\right)$ if $\chi_{j}(x) \neq 0$ and $\alpha_{j-1}(t, x) \in$ $\left.U_{j}\right)$;

$$
\rho\left(\alpha_{j-1}(t, x), \alpha_{j}(t, x)\right) \leq k l(x) \chi_{j}(x) t
$$

and

$$
\varphi\left(\alpha_{j}(t, x)\right)-\varphi\left(\alpha_{j-1}(t, x)\right) \leq-\varepsilon l(x) \chi_{j}(x) t .
$$

Indeed, for $j=1$, (4) follows immediately from (3). To show (5), let $x$ be any point in $U_{1}$, and consider the path $\sigma_{1}(s)=\alpha_{1}(s, x) \quad(0 \leq s \leq t)$ joining $x$ to $\alpha_{1}(t, x)$. We have

$$
\rho\left(x, \alpha_{1}(t, x)\right) \leq \int_{0}^{t}\left\|\sigma_{1}^{\prime}(s)\right\| d s \leq k \int_{0}^{t}\left\|\frac{d}{d s} f_{1}\left(\sigma_{1}(s)\right)\right\|_{x_{1}} d s=k l(x) \chi_{1}(x) t .
$$

To show (6), let $x \in U_{1}$ and use the mean value theorem to find $\theta \in(0,1)$ such that

$$
\begin{gathered}
\varphi\left(\alpha_{1}(t, x)\right)-\varphi(x)=\varphi \circ f_{1}^{-1}\left(f_{1}(x)-t l(x) \chi_{1}(x) \frac{v_{1}}{\left\|v_{1}\right\|}\right)-\varphi \circ f_{1}^{-1}\left(f_{1}(x)\right) \\
=\operatorname{tl}(x) \chi_{1}(x)\left\langle\left(\varphi \circ f_{1}^{-1}\right)^{\prime}\left(f_{1}(x)-\theta t l(x) \chi_{1}(x) \frac{v_{1}}{\left\|v_{1}\right\|}\right), \frac{v_{1}}{\left\|v_{1}\right\|}\right\rangle .
\end{gathered}
$$

It follows from (2), that if $x \in U_{1}$ then

$$
\varphi\left(\alpha_{1}(t, x)\right)-\varphi(x) \leq-\varepsilon l(x) \chi_{1}(x) t .
$$


The same trivially holds when $x \notin U_{1}$ since then $\chi_{1}(x)=0$.

Suppose now (4), (5), and (6) verified up to $j-1$, and let us prove them for $\alpha_{j}(t, x)$. First note that (5) and the triangular inequality give

$$
\rho\left(x, \alpha_{j-1}(t, x)\right) \leq k t l(x) \sum_{k=1}^{j-1} \chi_{k}(x) \leq k t .
$$

Since $t<\delta_{0} / 1+k^{2}$, we get that $\rho\left(x, \alpha_{j-1}(t, x)\right) \leq \delta_{0} / 2$. But this implies that for any $x \in \operatorname{supp}\left(\chi_{j}\right)$ with $\alpha_{j-1}(t, x) \in U_{j}$ we have

$$
\rho\left(x, \alpha_{j-1}(t, x)\right)=\inf \left\{L(\sigma) ; \sigma \text { joining } x \text { to } \alpha_{j-1}(t, x) \text { and } \sigma \subset U_{j}\right\},
$$

because if such a path $\sigma$ leaves $U_{j}$, we would have that $L(\sigma) \geq \delta_{0}$ in view of the fact that $\rho\left(x, X \backslash U_{j}\right) \geq \delta_{0}$. But if $\sigma \subset U_{j}$ joins $x$ to $\alpha_{j-1}(t, x)$ we have

$$
\begin{aligned}
L(\sigma) & =\int_{a}^{b}\left\|\sigma^{\prime}(s)\right\| d s \geq \frac{1}{k} \int_{a}^{b}\left\|\frac{d}{d s} f_{j}(\sigma(s))\right\|_{x_{j}} d s \\
& \geq \frac{1}{k}\left\|\int_{a}^{b} \frac{d}{d s} f_{j}(\sigma(s)) d s\right\|_{x_{j}}=\frac{1}{k}\left\|f_{j}\left(\alpha_{j-1}(t, x)\right)-f_{j}(x)\right\|_{x_{j}} .
\end{aligned}
$$

It follows that

$$
\left\|f_{j}\left(\alpha_{j-1}(t, x)\right)-f_{j}(x)\right\|_{x_{j}} \leq k \rho\left(x, \alpha_{j-1}(t, x)\right) \leq k^{2} t .
$$

Hence

$$
\left\|f_{j}\left(\alpha_{j-1}(t, x)\right)-t l(x) \chi_{j}(x) \frac{v_{j}}{\left\|v_{j}\right\|}-f_{j}(x)\right\|_{x_{j}} \leq k^{2} t+t<\delta_{0} .
$$

This implies that $f_{j}\left(\alpha_{j-1}(t, x)\right)-t l(x) \chi_{j}(x) v_{j} /\left\|v_{j}\right\| \in f_{j}\left(U_{j}\right)$ whenever $x \in$ $\operatorname{supp}\left(\chi_{j}\right)$ and $\alpha_{j-1}(t, x) \in U_{j}$, which clearly proves (4).

For $(5)$, consider the path $\sigma_{j}(s)=f_{j}^{-1}\left(f_{j}\left(\alpha_{j-1}(t, x)\right)-s l(x) \chi_{j}(x) v_{j} /\left\|v_{j}\right\|\right)$ for $0 \leq s \leq t$ and note that

$$
\rho\left(\alpha_{j-1}(t, x), \alpha_{j}(t, x)\right) \leq L\left(\sigma_{j}\right) \leq \int_{0}^{t}\left\|\sigma^{\prime}(s)\right\| d s \leq k l(x) \chi_{j}(x) t .
$$

Assertion (6) is also shown as in the case $j=1$ by using the mean value theorem between $\alpha_{j-1}(t, x)$ and $\alpha_{j}(t, x)$. The induction is complete.

Now set $\alpha(t, x)=\alpha_{m}(t, x)$ and $g(x)=l(x) \sum_{j=1}^{m} \chi_{j}(x)$. They clearly verify the properties claimed in the lemma.

Proof of Theorem 1. Let $0<\varepsilon$, and consider a set $A$ in $\mathscr{F}$ such that

$$
c \leq \sup \varphi(A)<c+\varepsilon^{2} / 8 \text {. }
$$

We shall prove the existence of $x_{\varepsilon} \in X \backslash B$ such that

(i) $c-\varepsilon^{2} / 8 \leq \varphi\left(x_{\varepsilon}\right) \leq c+5 \varepsilon^{2} / 4$;

(ii) $\operatorname{dist}\left(x_{\varepsilon}, \bar{F}\right) \leq 3 \varepsilon / 2$;

(iii) $\left\|d \varphi\left(x_{\varepsilon}\right)\right\| \leq 10 \varepsilon$;

(iv) $\operatorname{dist}\left(x_{\varepsilon}, A\right) \leq \varepsilon / 2$. 
Let $F_{\varepsilon}=\{x \in X ; \operatorname{dist}(x, F)<\varepsilon\}$, and consider the subspace $\mathscr{L}$ of $C([0,1] \times X ; X)$ consisting of all maps $\eta$ such that

$$
\eta(t, x)=x \quad \text { for all }(t, x) \in K_{0}=(\{0\} \times X) \cup\left([0,1] \times\left(A \backslash F_{\varepsilon}\right) \cup B\right)
$$

and $\sup \{\rho(\eta(t, x), x) ; t \in[0,1], x \in X\}<+\infty$. Since $(\{0\} \times X) \cup([0,1] \times$ $B) \subset K_{0}$, we get that $\eta(\{1\} \times A) \in \mathscr{F}$ for all $\eta$ in $\mathscr{L}$.

The space $\mathscr{L}$ equipped with the uniform metric $\delta$ is a complete metric space. Now consider the following perturbations of $\varphi$,

$$
\begin{aligned}
& \varphi_{1}(x)=\max \left\{0, \varepsilon^{2}-\varepsilon \operatorname{dist}(x, F)\right\}, \\
& \varphi_{2}(x)=\min \left\{\varepsilon^{2} / 8, \quad \varepsilon \operatorname{dist}\left(x,\left(A \backslash F_{\varepsilon}\right) \cup B\right)\right\} .
\end{aligned}
$$

Denote by $\psi$ the function $\varphi+\varphi_{1}+\varphi_{2}$, by $K_{1}$ the set $\{1\} \times A$, and define a lower semicontinuous function $I: \mathscr{L} \rightarrow \mathbf{R}$ by

$$
I(\eta)=\sup \{\psi(\eta(1, x)) ; x \in A\}=\sup \psi\left(\eta\left(K_{1}\right)\right) .
$$

Let $d=\inf \{I(\eta) ; \eta \in \mathscr{L}\}$, and note that since $\eta\left(K_{1}\right) \in \mathscr{F}$ for all $\eta \in \mathscr{L}$ and since $\varphi_{1}=\varepsilon^{2}$ on $F$, we get from conditions $\left(F^{\prime} 1\right)$ and $\left(F^{\prime} 2\right)$ that

$$
I(\eta) \geq \sup \left\{\left(\varphi+\varphi_{1}\right)(x) ; x \in \eta\left(K_{1}\right) \cap F\right\} \geq c+\varepsilon^{2} .
$$

Hence

$$
d \geq c+\varepsilon^{2}
$$

Consider now the identity function $\bar{\eta}$ in $\mathscr{L}$ defined by $\bar{\eta}(t, x)=x$ for all $(t, x)$ in $[0,1] \times X$, and note that

(11) $d \leq I(\bar{\eta})=\sup \left\{\left(\varphi+\varphi_{1}+\varphi_{2}\right)(x) ; x \in A\right\}<c+\varepsilon^{2} / 8+\varepsilon^{2}+\varepsilon^{2} / 8=c+5 \varepsilon^{2} / 4$.

Combine (10) and (11) to get that $\bar{\eta}$ is an element in $\mathscr{L}$ verifying

$$
I(\bar{\eta})<c+5 \varepsilon^{2} / 4 \leq d+\varepsilon^{2} / 4=\inf \{I(\eta) ; \eta \in \mathscr{L}\}+\varepsilon^{2} / 4 .
$$

Apply Ekeland's theorem ([AE] Corollary 5.3.2) to get $\eta_{0}$ in $\mathscr{L}$ such that

$$
\begin{gathered}
I\left(\eta_{0}\right) \leq I(\bar{\eta}), \\
\delta\left(\eta_{0}, \bar{\eta}\right) \leq \varepsilon / 2, \\
I(\eta) \geq I\left(\eta_{0}\right)-(\varepsilon / 2) \delta\left(\eta, \eta_{0}\right) \text { for all } \eta \text { in } \mathscr{L} .
\end{gathered}
$$

Let $C=\left\{x \in \eta_{0}\left(K_{1}\right) ; \psi(x)=\max \psi\left(\eta_{0}\left(K_{1}\right)\right\}\right.$ and $B^{\prime}=\left(A \backslash F_{\varepsilon}\right) \cup B$. We need to show that

$$
C \cap B^{\prime}=\varnothing \text {. }
$$

For that we shall prove that for some $\tau>0$,

$$
\sup \psi\left(\left(A \backslash F_{\varepsilon}\right) \cup B\right) \leq \max \psi\left(\eta_{0}\left(K_{1}\right)\right)-\tau .
$$

Indeed, by $\left(\mathrm{F}^{\prime} 1\right)$ there exists $x_{0} \in M\left(\eta_{0}\left(K_{1}\right) \cap F ; \varphi\right) \backslash B$. Hence

$$
\max \psi\left(\eta_{0}\left(K_{1}\right)\right) \geq \max \psi\left(\eta_{0}\left(K_{1}\right) \cap F\right) \geq c+\varepsilon^{2}+\varphi_{2}\left(x_{0}\right)
$$

Note that $\tau=\varphi_{2}\left(x_{0}\right)>0$ since $x_{0} \notin B \cup\left(A \backslash F_{\varepsilon}\right)$. On the other hand since $\varphi_{1}=0$ outside $F_{\varepsilon}$ and $\varphi_{2}=0$ on $A \backslash F_{\varepsilon}$, we get from (1) that

$$
\sup \psi\left(A \backslash F_{\varepsilon}\right) \leq \sup \varphi(A)<c+\varepsilon^{2} / 8 \text {. }
$$


Moreover, since $\sup \varphi(B) \leq c$, we have

$$
\sup \psi\left(B \cap F_{\varepsilon}\right)=\sup \left(\varphi+\varphi_{1}\right)\left(B \cap F_{\varepsilon}\right) \leq c+\varepsilon^{2} .
$$

The last three displayed inequalities clearly imply (17).

We shall now prove the following

Claim. There exists $x_{\varepsilon} \in C=\eta_{0}(M)$ such that $\left\|d \varphi\left(x_{\varepsilon}\right)\right\| \leq 10 \varepsilon$.

Before proving it, let us show how it implies Theorem 1. First note that since $x_{\varepsilon} \in C$, we have by (13) and (11) that $d \leq\left(\varphi+\varphi_{1}+\varphi_{2}\right)\left(x_{\varepsilon}\right) \leq c+5 \varepsilon^{2} / 4$. Since $0 \leq \varphi_{1} \leq \varepsilon^{2}$ and $0 \leq \varphi_{2} \leq \varepsilon^{2} / 8$, we get from (10) that $c-\varepsilon^{2} / 8 \leq \varphi\left(x_{\varepsilon}\right) \leq$ $c^{\prime}+5 \varepsilon^{2} / 4$, which is assertion $(\mathrm{i})$.

For (ii) write $x_{\varepsilon}=\eta_{0}(1, x)$ where, in view of (16), $x$ is necessarily in $F_{\varepsilon}$. Hence $\operatorname{dist}(x, F) \leq \varepsilon$. On the other hand, by $(14)$ we have $\rho\left(x_{\varepsilon}, x\right)=$ $\rho\left(\eta_{0}(1, x), x\right) \leq \delta\left(\eta_{0}, \bar{\eta}\right) \leq \varepsilon / 2$. Hence $\operatorname{dist}\left(x_{\varepsilon}, F\right) \leq 3 \varepsilon / 2$. Note finally that (iv) is satisfied since $x \in A$.

Back to the claim. Suppose it is false. Fix $1<k<2$ and apply Lemma 1 to the sets $C$ and $B^{\prime}$ to get $\alpha(t, x)$ verifying the conclusion of Lemma 1 with a suitable function $g$ and a time $t_{0}>0$.

For $0<\lambda<t_{0}$, consider the function $\eta_{\lambda}(t, x)=\alpha\left(t \lambda, \eta_{0}(t, x)\right)$. It belongs to $\mathscr{L}$, since it is clearly continuous on $[0,1] \times X$ and since for al $(t, x) \in$ $(\{0\} \times X) \cup\left([0,1] \times B^{\prime}\right)$, we have $\eta_{\lambda}(t, x)=\alpha\left(t \lambda, \eta_{0}(t, x)\right)=\alpha(t \lambda, x)=x$.

Since $\delta\left(\eta_{\lambda}, \eta_{0}\right)<k t \lambda \leq k \lambda$, we get from (15) that $I\left(\eta_{\lambda}\right) \geq I\left(\eta_{0}\right)-\varepsilon k \lambda / 2$. Since $A$ is compact, let $x_{\lambda} \in A$ be such that $\psi\left(\eta_{\lambda}\left(1, x_{\lambda}\right)\right)=I\left(\eta_{\lambda}\right)$. We have

$$
\psi\left(\eta_{\lambda}\left(1, x_{\lambda}\right)\right)-\psi\left(\eta_{0}(1, x)\right) \geq-\varepsilon k \lambda / 2 \text { for every } x \in A \text {. }
$$

Since the Lipschitz constants of both $\varphi_{1}$ and $\varphi_{2}$ are less than $\varepsilon$, we get

$$
\varphi\left(\eta_{\lambda}\left(1, x_{\lambda}\right)\right)-\varphi\left(\eta_{0}\left(1, x_{\lambda}\right)\right) \geq-5 \varepsilon \lambda k / 2
$$

On the other hand, by Lemma 1 (iii) we have for all $x$

$$
\begin{aligned}
\varphi\left(\eta_{\lambda}\left(1, x_{\lambda}\right)\right)-\varphi\left(\eta_{0}\left(1, x_{\lambda}\right)\right) & =\varphi\left(\alpha\left(\lambda, \eta_{0}\left(1, x_{\lambda}\right)\right)\right)-\varphi\left(\eta_{0}\left(1, x_{\lambda}\right)\right) \\
& \leq-5 \varepsilon \lambda g\left(\eta_{0}\left(1, x_{\lambda}\right)\right) .
\end{aligned}
$$

Combining (19) and (20) we get

$$
-5 \varepsilon k / 2 \leq-5 \varepsilon g\left(\eta_{0}\left(1, x_{\lambda}\right)\right) .
$$

If now $x_{0}$ is any cluster point of $\left(x_{\lambda}\right)$ when $\lambda \rightarrow 0$, we have from (18) that $\eta_{0}\left(1, x_{0}\right) \in C$ and hence $g\left(\eta_{0}\left(1, x_{0}\right)\right)=1$. Since $k<2$, this clearly contradicts $(21)$ and therefore the initial claim was true. The proof of the theorem is complete.

\section{THE EQUIVARIANT CASE}

Suppose $G$ is a compact Lie group acting differentiably on the manifold $X$, and suppose $\varphi$ is invariant under the action of $G$. We then obtain the same conclusion as in Theorem 1 , provided the class $\mathscr{F}$ is stable under those homotopies that are $G$-equivariant while preserving the boundary $B$. The proofs consist of equivariant adaptations of the above arguments, and we leave them for the interested reader. 
Suppose now Ind $_{G}$ is a topological index associated to $G$. that is $\operatorname{Ind}_{G}$ is a mapping from the closed $G$-invariant subsets of $X$ into $\mathbf{N} \cup\{+\infty\}$ verifying the following properties:

(I1) $\operatorname{Ind}_{G}(A)=0$ if and only if $A=\varnothing$.

(I2) $\operatorname{Ind}_{G}\left(A_{2}\right) \geq \operatorname{Ind}_{G}\left(A_{1}\right)$ if there is a $G$-equivariant continuous map from $A_{1}$ to $A_{2}$.

(I3) If $K$ is compact invariant, there exists a closed invariant neighborhood $K^{\delta}=\{x ; \operatorname{dist}(x, K) \leq \delta\}$ of $K$ so that $\operatorname{Ind}_{G}\left(K^{\delta}\right)=\operatorname{Ind}_{G}(K)$.

(I4) $\operatorname{Ind}_{G}\left(A_{1} \cup A_{2}\right) \leq \operatorname{Ind}_{G}\left(A_{1}\right)+\operatorname{Ind}_{G}\left(A_{2}\right)$ for all invariant subsets $A_{1}, A_{2}$.

For each $n$, consider the class of sets

$$
\Sigma_{n}^{B}=\left\{A ; A \text { compact invariant containing } B \text { and } \operatorname{Ind}_{G}(A) \geq n\right\} .
$$

It is clearly $G$-homotopy stable with boundary $B$. The following result is well known when $B=\varnothing$ or when $\sup \varphi(B)<c$.

Proposition 1. Let $\varphi$ be a $G$-invariant $C^{1}$-functional on $X$, and consider $a G$ homotopy stable family $\mathscr{F}$ with a closed boundary $B$. Set $c=c(\varphi, \mathscr{F})$ and assume $\varphi$ verifying $(P S)_{c}$. If $c=c(\varphi, \mathscr{F})=c\left(\varphi, \sum_{m}^{B}\right)$ for some $m \geq 1$, then we have

$$
\operatorname{Ind}_{G}\left(\overline{K_{c} \backslash B}\right) \geq \inf \left\{\operatorname{Ind}_{G}(A) ; A \in \mathscr{F}\right\}-\operatorname{Ind}_{G}(B \cap\{\varphi \geq c\})-m+1 .
$$

In particular, if $\mathscr{F}=\sum_{m+p}^{B}$ then

$$
\operatorname{Ind}_{G}\left(\overline{K_{c} \backslash B}\right) \geq p+1-\operatorname{Ind}_{G}(B \cap\{\varphi \geq c\}) .
$$

Proof. Let $U$ be an open invariant neighborhood of $\overline{K_{c} \backslash B}$ with the same index. Also let $V$ be an open invariant neighborhood of $B \cap\{\varphi \geq c\}$ with the same index. If the assertion were false, then we get that for any $A \in \mathscr{F}$, the set $(A \backslash U) \backslash V \in \sum_{m}^{B}$. This means that the set $F=(\{\varphi \geq c\} \backslash U) \backslash V$ and $B$ verify conditions $\left(\mathrm{F}^{\prime} 1\right)$ and $\left(\mathrm{F}^{\prime} 2\right)$ with respect to the class $\mathscr{F}$. By the equivariant version of Theorem 1.bis, $F \cap K_{c} \neq \varnothing$, which is a contradiction.

\section{REFERENCES}

[AE] J. P. Aubin and I. Ekeland, Applied non-linear analysis, Pure Appl. Math, Wiley, New York, 1984.

[BN] H. Brezis and L. Nirenberg, Remarks on finding critical points, preprint, 1990.

[GP] N. Ghoussoub and D. Preiss, A general mountain pass principle for locating and classifying critical points, Ann. Inst. H. Poincaré Anal. Non Linéaire 6 (1989), 321-330.

[G1] N. Ghoussoub, Location, multiplicity and Morse Indices of min-max critical points, J. Reine Angew. Math. 417 (1991), 27-76.

[G2] N. Ghoussoub, New aspects of the calculus of variations in the large and applications to differential equations, monograph, 1991 (to appear).

[P] R. Palais, Lusternik-Schnirelman theory on Banach manifolds, Topology 5 (1966), 115-132.

[R] P. H. Rabinowitz, Minimax methods in critical point theory with applications to differential equations, CBMS, No. 65, Amer. Math. Soc., Providence, RI, 1986.

[S] A. Szulkin, Ljusternik-Schnirelmann theory on $C^{1}$-manifold, Ann. Inst. H. Poincaré Analyse non linéaire, Vol. 5, 1988, pp. 119-139.

Department of Mathematics, The University of British Columbia, Vancouver, Canada V6T 1 Y4 